\title{
HBV reactivation under immunosuppressive treatment - a case series
}

\author{
Mihaela Rădulescu ${ }^{1,2}$, Anca-Ruxandra Negru ${ }^{1 *}$, Cristina Popescu ${ }^{1,2}$, Violeta Molagic ${ }^{1}$, Daniela Munteanu1, \\ Raluca Mihăilescu', Cătălin Tilişcan 1,2, Alina Lobodan', Irina Lăpădat', Smaranda Gliga', Georgiana Jugănaru', \\ Aida Adamescu², Victoria Aramă ${ }^{1,2}$ \\ From The 10th Edition of the Scientific Days of the National Institute for Infectious Diseases "Prof Dr Matei \\ Bals" \\ Bucharest, Romania. 15-17 October 2014
}

\section{Background}

Inactive HBV carriers, under immunosuppressive treatment for malignancies or rheumatologic diseases, have an increased risk for HBV reactivation. HBV reactivation under immunosuppression has a high rate of acute liver failure and death. HBV screening is mandatory for patients with hematological malignancies or rheumatologic diseases who are due to receive immunosuppressive treatment.

\section{Methods}

We retrospectively analyzed 13 patients from Department 3 of the National Institute for Infectious Diseases "Prof. Dr. Matei Balş" that were diagnosed with HBV reactivation under immunosuppressive treatment for different malignancies or rheumatologic diseases.

\section{Results}

Thirteen patients were enrolled, 6 women and 7 men, with a median age of 56 years [32-70.5]. Seven patients were diagnosed with hematologic malignancies (6 with non-Hodgkin lymphoma and 1 with chronic lymphatic leukemia), 4 with rheumatologic diseases (1 with ankylosing spondylitis, 1 with Reiter syndrome and 2 with rheumatoid polyarthritis), 1 patient with ovarian cancer and 1 patient with renal transplant. Of 13 patients, one had negative HBsAg and protective HBs antibodies titers, before immunosuppressive treatment, with further HBs retroseroconversion.

\footnotetext{
* Correspondence: anca1808@yahoo.com

${ }^{1}$ National Institute for Infectious Diseases "Prof. Dr. Matei Balş", Bucharest, Romania

Full list of author information is available at the end of the article
}

On admission, TGP median value was $1,125 \mathrm{IU} / \mathrm{dL}$ [491-1738]. Median prothrombin concentration on admission was $85 \%$ [68-101] and median nadir of prothrombin concentration was $66 \%$ [54-84].

Median hospitalization period was 20.77 days. Hospitalization period was directly correlated with ALT and AST values at the time of admission $(\mathrm{p}=0.06$ respectively $\mathrm{p}=$ 0.015 ) and negatively correlated with prothrombin concentration at admission $(\mathrm{p}=0.059)$ and lymphocytes number $(\mathrm{p}=0.058)$.

Ten patients were treated with entecavir and three with lamivudine. Three patients died, all of them with hematologic malignancies - two deaths were due to hematologic disease and one due to liver failure. Median age of deceased patients was 73 years [68.0-75.0], while in the surviving group the median age was 50 years [27.7-58.5] $(\mathrm{p}=0.049)$.

\section{Conclusion}

In HBV reactivation patients, older age is a risk factor for mortality. Higher liver transaminases and lower prothrombin concentration and lymphocytes are associated with longer hospitalization period. Even patients with negative HBs antigen can reactivate HBV infection during immunosuppression, requiring close monitoring.

\footnotetext{
Authors' details

${ }^{1}$ National Institute for Infectious Diseases "Prof. Dr. Matei Balş", Bucharest, Romania. ${ }^{2}$ Carol Davila University of Medicine and Pharmacy, Bucharest, Romania.
}

Published: 15 October 2014 
doi:10.1186/1471-2334-14-S7-011

Cite this article as: Rădulescu et al.: HBV reactivation under

immunosuppressive treatment - a case series. BMC Infectious Diseases

2014 14(Suppl 7):011.

Submit your next manuscript to BioMed Central and take full advantage of:

- Convenient online submission

- Thorough peer review

- No space constraints or color figure charges

- Immediate publication on acceptance

- Inclusion in PubMed, CAS, Scopus and Google Scholar

- Research which is freely available for redistribution

Submit your manuscript at 